\title{
Multi-Timescale Power Management for Islanded Microgrids Including Storage and Demand Response
}

\section{Authors: S.A. Pourmousavi, M.H. Nehrir, \& R.K. Sharma}

This is a postprint of an article that originally appeared in IEEE Transactions on Smart Grid in May 2015. (c) 2015 IEEE. Personal use of this material is permitted. Permission from IEEE must be obtained for all other users, including reprinting/ republishing this material for advertising or promotional purposes, creating new collective works for resale or redistribution to servers or lists, or reuse of any copyrighted components of this work in other works.

Pourmousavi, S.A., M.H. Nehrir, and R.K. Sharma. "Multi-Timescale Power Management for Islanded Microgrids Including Storage and Demand Response." IEEE Transactions on Smart Grid 6, no. 3 (May 2015): 1185-1195. DOI: 10.1109/TSG.2014.2387068.

Made available through Montana State University's ScholarWorks scholarworks. montana.edu 


\title{
Multi-Timescale Power Management for Islanded Microgrids Including Storage and Demand Response
}

\author{
S. Ali Pourmousavi, Student Member, IEEE, M. Hashem Nehrir, Life Fellow, IEEE, Ratnesh K. \\ Sharma, Member, IEEE
}

\begin{abstract}
Power management is an essential tool for microgrid (MG) safe and economic operation, particularly in the islanded operation mode. In this study, a multi-timescale costeffective power management algorithm is proposed for islanded MG operation targeting generation, storage, and demand management. Comprehensive modeling, cost, and emission calculations of the MG components are developed in this study to facilitate high accuracy management. While the MG's overall power management and operation is carried out every several minutes to hours, depending on the availability of the required data, simulation for highly dynamic devices (such as batteries and electric water heaters (EWHs) used for demand response (DR)) are performed every minute. This structure allows accurate, scalable, and practical power management taking into consideration the intra-interval dynamics of battery and EWHs. Two different ON/OFF strategies for EWH control are also proposed for DR application. Then, the power management algorithm is implemented using the two different DR strategies and the results are compared with the no-DR case. Actual solar irradiation, ambient temperature, non-EWH load demand, and hot water consumption data are employed in the simulation studies. The simulation results for the MG studied show the effectiveness of the proposed algorithm to reduce both MG's cost and emission.
\end{abstract}

Index Terms-Demand response, diesel generator efficiency and emission model, battery dynamic modeling, power management, electric water heater dynamic model.

\section{INTRODUCTION}

A CCORDING to the U.S. department of Energy's (DOE's) list of five fundamental technologies that will drive the smart grid [1], "advanced control methods to observe essential components for rapid diagnosis and precise solutions to events" is envisioned as one of the most critical apparatus for successful implementation of smart grid. Given the complexity of the smart grid implementation, MGs have

This work was supported by NEC Labs America, and the U.S. Department of Energy, Office of Science, Basic Energy Sciences, under Award \# DEFG02-11ER46817.

S.A. Pourmousavi (email: s.pourmousavikani@msu.montana.edu), and M.H. Nehrir (email: hnehrir@ece.montana.edu) are with the Electrical and Computer Engineering Department, Montana State University, Bozeman, 59717 USA, and Ratnesh K. Sharma (e-mail: ratnesh@sv.nec-labs.com), is with NEC Laboratories America, Cupertino CA 95014 USA. emerged as a bridging technology to pave the way to the smart grid [2]. The key to wider recognition and employment of MGs is innovative power management strategy [3].

In general, real-time MG management is classified into energy and power management. The energy management algorithms deal with monitoring and operation of a complex system of electrical, thermal, and mechanical components with emphasis on desired and longer term outcomes. However, the objective of power management is to affect the instantaneous operational conditions towards certain desired performance [4]-[11]. From a general perspective, both power and energy management refer to control actions that are based on particular objectives.

MG management algorithms can be divided into centralized [4]-[9] and decentralized architecture [10], [11]. Structurally, MGs can be operated in grid-tied [4], [7], [9]-[11], islanded [5], [6], or a combination of both modes, e.g., [8]; each of the above modes has certain requirements to be fulfilled. This paper focuses on the design, modeling, and proposing a comprehensive cost-effective rule-based and multi-timescale power management algorithm for an islanded MG that operates independently in a remote area. Although rule-based algorithms may not be ideal for grid-tied and plug-and-play operation of MGs, they are ideal in real-world applications with minimum computational burden, in particular for rural areas. In this study, the MG components include a diesel generator (DiG), solar PV panels, and storage batteries, which are commonly available for residential applications.

In islanded MGs, ancillary services is provided using storage and DR to compensate for renewable generation and load demand variability. Although the DR is foreseen to be an essential part of MGs' cost-effective operation, some literature on power management do not include the DR in their proposed algorithm, e.g. [6]-[8], and others consider generic load for DR, e.g. [5], [10], [11]. However, in this study, DR is implemented with EWHs for which a dynamic model with actual hot water consumption data is used. Although EWH is used as an example to show the effectiveness of DR in the proposed management algorithm, it can be replaced with any other responsive load that has energy storage capability, such as electric space heating and cooling. Two different ON/OFF control algorithms (called DR.A and DR.B) are introduced and 
implemented to provide the required increase or decrease in load at the time needed.

Moreover, the multi-timescale structure of the proposed algorithm allows two different simulation intervals for $\mathrm{MG}$ components based on their dynamic behavior and the available data. This way, simulation interval of highly dynamic components (e.g., battery and EWHs) will be decoupled from those of the available load and generation data, which might be available with different intervals (e.g., 20 minutes or 1 hour). This way, the flexibility and accuracy of the algorithm will be increased, which can result in faster and more accurate control of MGs.

The major contributions of this paper are: The multitimescale structure, the cost-effective operation in rule-based power management structure, and the DR application. We also included detailed dynamic models of the battery and EWH (that we have developed previously), [12], [13]-[15], in our proposed multi-timescale power management algorithm. MATLAB $^{\circledR}$ GUIs have been developed to easily scale and modify the proposed MG structure and simulation time interval as well as to observe the MG performance through a monitoring GUI during the simulation.

The rest of the paper is organized as follows. MG design and modeling are briefly explained in Section II, including an overall view of the proposed simulation environment. Section III presents the proposed power management algorithm and two different DR strategies for EWHs. Simulation results and discussion are presented in Section IV, and the paper is concluded in Section V.

\section{MG DESIGN AND MoDELLING}

In the following sub-sections, MG design and comprehensive modeling are presented for the MG's components, including solar PV, DiG, storage, and EWH loads.

\section{A. MG Design:}

The proposed islanded MG (Fig. 1) is designed for a residential neighborhood including 100 houses. The residential units are assumed to have EWHs, which are used for the DR (when needed) to provide ancillary services for the MG. MG design (unit sizing) is carried out using HOMER ${ }^{\circledR}$ [16]. Actual minute-by-minute residential load data, recorded for a single house between 2006 to 2010, has been used [17]. The load demand data for 100 houses is generated by a series of random numbers with an average of $0.1 \mathrm{~kW}$ and standard deviation of 0.2 , added to the one-house data available to us. Actual solar irradiation and ambient temperature have also been used [9]. It is assumed that all PV arrays experience similar solar irradiation and ambient temperature. The component sizes for the application obtained from HOMER are: $570 \mathrm{~kW}$ of PV, $350 \mathrm{~kW}$ of DiG, and $840 \mathrm{kWh}$ of battery.

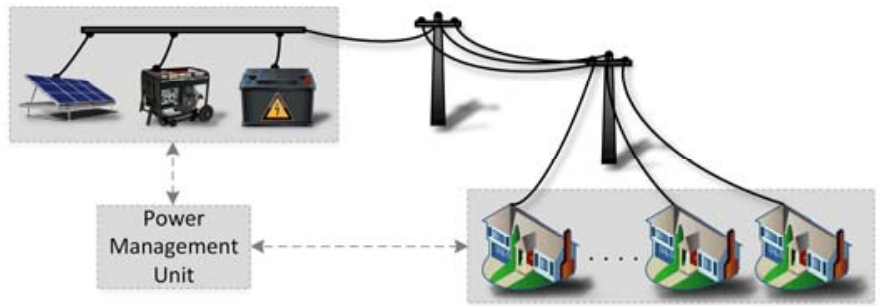

Fig. 1. Schematic diagram of the MG configuration.

\section{B. MG modeling:}

Comprehensive modeling of the MG components is an essential part of the proposed multi-timescale structure to perform accurate simulation and decisions making. In this sub-section, model of each component will be briefly explained in addition to the cost and emission calculations that were applied for the relevant components.

\section{PV Model}

In this study, a comprehensive mathematical model of PV, [18], is used where the PV operating point is calculated based on solar irradiation and ambient temperature. An actual grid wattage PV module from SunPower Corporation (SPR-200BLK), which is suitable for residential applications, was selected for modeling [19]. The maximum power point (MMP) of the PV module at different solar irradiation and ambient temperatures is calculated using the first derivative of the module instantaneous power with respect to the module's voltage. In order to avoid solving numerical nonlinear equation in each iteration, the MPP corresponding to different common solar irradiation levels and ambient temperature were calculated and stored in a 2-D lookup table for future use in the power management algorithm (PMA). This procedure simplifies the implementation of the proposed PMA for realworld application. A $100-\mathrm{W} / \mathrm{m}^{2}$ solar irradiation interval and $3^{\circ} \mathrm{C}$ temperature interval is used in the 2-D lookup table. These intervals are small enough for precise linear approximation between two neighboring sample points. This has been verified through sensitivity analyses for different solar irradiation values and ambient temperatures, which are not given in this paper because of page limit.

Since the PV system is connected to the MG through $\mathrm{DC} / \mathrm{DC}$ and DC/AC converters, the efficiency of converters has also been modeled as a function of the normalized input power [7]. Since converters' efficiency drops rapidly for small loading, a deadband of $5 \%$ of the module nominal power (i.e., $200 \mathrm{~W}$ ) was considered for PV operation.

In addition to the model of the MG components, their accurate operational cost calculation is also essential for an accurate PMA. The operational cost is commonly divided into ownership and operating costs [20]. In order to calculate the ownership cost of MG components, a general framework considering uncertainty and risk analyses was used, which has been proposed by the authors in [21]. However, no ownership cost is considered for the PV system because PV modules usually last for 20-25 years, equal or more than the 20-year project lifetime assumed. Therefore, no replacement cost is required throughout the project lifetime. Also, PV operating 
cost (which only comprises maintenance cost) is very cheap, less than $0.005 \$ / \mathrm{kWh}[22]$. Therefore, it is reasonable to consider PV power as negative load to extract its maximum available power at any time.

\section{DiG Model}

Since DiGs are equipped with electronic speed governor and automatic voltage regulator (AVR), their dynamic behavior is not a concern as long as the simulation interval does not go below one minute. The DiG selected for this study is rated at $350 \mathrm{~kW}$ (CumminsOnan Commercial DFEGC350D6 diesel genset) with 1.5 and 2.1 seconds recovery time for voltage and frequency, respectively, after $100 \%$ change in load [23]. In addition, DiGs have incredibly fast cold startup time (about 10 seconds for DiGs smaller than $1000 \mathrm{~kW}$ ) [24]. Therefore, the DiG modeling for this study is limited to its cost and emission. Fuel consumption data for the DiG used in this study are given in Table I. It should be noted that DiGs are usually responsible to establish frequency in an islanded MG, which requires the DiG to operate in standby mode at all times. Therefore, the DiG will consume a minimum amount of fuel $(15 \mathrm{~L} /$ hour $)$ at no load.

TABLE I

ACTUAL FUEL CONSUMPTION FOR THE DiG STUdied [23].

\begin{tabular}{l|c|c|c|c|c}
\hline Model C350D6 & $\begin{array}{c}\text { No } \\
\text { load }\end{array}$ & $\begin{array}{c}25 \% \\
\text { load }\end{array}$ & $\begin{array}{c}50 \% \\
\text { load }\end{array}$ & $\begin{array}{c}75 \% \\
\text { load }\end{array}$ & $\begin{array}{c}100 \% \\
\text { load }\end{array}$ \\
\hline Fuel Consumption (L/hour) & 15 & 30.2 & 50.4 & 72 & 96 \\
\hline
\end{tabular}

Based on the approach reported in [21], the ownership cost for the DiG under consideration is calculated as 1.6018 $\$$ /hour. The DiG operating cost consists of fuel cost, cost of other regularly replaceable components, and overhaul costs. Fuel consumption data for a variety of DiGs ranging from 6 to $2260 \mathrm{~kW}$ are compiled and modeled in [10], and is utilized in HOMER $^{\circledR}$ [16]. However, employing a similar linear model for the DiG studied does not show an acceptable fit to the data given in Table I. A quadratic polynomial curve fitting (1) is tried on the data using MATLAB/Curve Fitting toolbox [25], and it gives an excellent fit to the data:

$$
F_{D i G}(i)=0.0001773 \mathrm{P}_{D i G}^{2}(i)+0.1709 \mathrm{P}_{D i G}(i)+14.67
$$

where $\mathrm{P}_{D i G}(i)$ is the DiG operating power level $(\mathrm{kW})$ at interval $i$. The cost of diesel is considered to be $\$ 0.97 / \mathrm{L}$ in this study. Comparison of the two methods (i.e., linear vs. quadratic curves) yields substantial differences which can result in erroneous fuel and cost calculations which can result in a series of incorrect decisions for the MG operation.

Maintenance cost is another component of the DiG's operating cost which has been ignored in some studies, e.g. [26]. Since the non-fuel operating cost data was not available for the specific DiG studied, the non-fuel operating cost data for 1-10 MW DiGs reported by EPRI, [27], is utilized. The total maintenance cost (i.e., operating cost minus fuel cost) is approximated as $\$ 75.44 / \mathrm{kW}$-installed by looking at the 20 year net present value of costs [27]. Therefore, annual maintenance cost of the $350-\mathrm{kW}$ DiG used in this study can be approximated as $\$ 1320$ year. This cost is for all maintenance levels and overhaul of the DiG used.
There is no emission data provided for this specific $350-\mathrm{kW}$ DiG. However, an extensive emission measurement has been carried out for $10-\mathrm{kW}$ DiGs used in residential applications and on military bases, reported in [28] for $10 \mathrm{~kW}$ DiGs [28]. In this reference, several experiments are carried out on three different 10-kW DiGs from the manufacturer of the DiG used in this study, and $\mathrm{CO}_{2}, \mathrm{CO}$, Ethylene, $\mathrm{NO}_{2}, \mathrm{NO}$, and hydrocarbon $(\mathrm{HC})$ production are reported. Since $\mathrm{CO}$ and $\mathrm{CO}_{2}$ typically account for more than $99 \%$ of the carbon emitted in engine exhaust [28], the emission production $\left(E M_{D G}\right)$ is categorized into $\mathrm{CO}_{2}$ and non- $\mathrm{CO}_{2}$ emissions, i.e., non- $\mathrm{CO}_{2}$ emissions are combined to simplify the overall emission equation. A fifth-order polynomial is found to be a good fit for the data as follows:

$$
\begin{aligned}
E M_{D i G}^{j}(i) & =a_{1}^{j} \cdot P_{D i G}^{5}(i)+a_{2}^{j} \cdot P_{D i G}^{4}(i)+a_{3}^{j} \cdot P_{D i G}^{3}(i) \\
& +a_{4}^{j} \cdot P_{D i G}^{2}(i)+a_{5}^{j} \cdot P_{D i G}(i)+a_{6}^{j}
\end{aligned}
$$

where $j$ is the type of emission at interval $i$. Coefficients for the emission types are given in Table II. The emission values for the $350-\mathrm{kW}$ DiG used in this study is approximated by linearly scaling up all values from the $10-\mathrm{kW}$ DiG by multiplying by 35 . This is only an approximation since no emission data for the $350-\mathrm{kW}$ DiG used was not available.

TABLE II

CURVE-FITTED COEFFICIENTS OF THE FUNCTIONS FOR EMISSION PRODUCTION

\begin{tabular}{l|c|c|c|c|c|c}
\hline Type & $a_{1}^{j}$ & $a_{2}^{j}$ & $a_{3}^{j}$ & $a_{4}^{j}$ & $a_{5}^{j}$ & $a_{6}^{j}$ \\
\hline $\mathrm{CO}_{2}$ & 1.511 & -35.47 & 290.1 & -1011 & 1519 & 1422 \\
\hline Non- $\mathrm{CO}_{2}$ & -0.01859 & 0.4579 & -4.128 & 17.959 & -43.776 & 105.9 \\
\hline
\end{tabular}

The total cost of emission, which is a function of fuel consumption, can be calculated as follows:

$$
C_{D i G}^{j}(i)=C^{j} \cdot E M_{D i G}^{j}(i) \cdot F_{D i G}(i) \times \frac{0.832}{1000}
$$

where $C^{j}$ is the damage cost (externality cost) of pollutant $j$, and $\frac{0.832}{1000}$ is the conversion factor for $\frac{1 \mathrm{~kg} \text { of fuel }}{\text { liter }} \times \frac{\mathrm{gr}}{\mathrm{kg}}$. The damage costs of $\mathrm{CO}_{2}$ is estimated as $\$ 0.014 / \mathrm{kg}$ in [29]. The cost for non- $\mathrm{CO}_{2}$ pollutants is taken from [30], which is estimated at $\$ 4.2 / \mathrm{kg}$ pollutant (total hydrocarbon) since they are the most dangerous pollutant for living organs' health. The DiG's efficiency can be calculated as follows [10]:

$$
\eta_{D i G}=\frac{P_{o u t}}{P_{i n}}=\frac{3600 \times P_{D i G}(i)}{F_{D i G}(i) \cdot E_{c}}
$$

where $E_{c}$ is the diesel energy density $(35000 \mathrm{~kJ} / \mathrm{L})$. The efficiency values for the linear and quadratic fuel consumption functions are shown in Fig. 2. The significant difference between the two curves can be due to the two different methods (i.e., linear and quadratic functions) used for fuel consumption calculation.

\section{Battery Model}

In this paper, a commonly used valve-regulated lead-acid (VRLA) battery dynamic model, described in [31] (also used in our earlier work for power/energy management studies [12]), is used. The VRLA model is slightly modified for this paper to work based on its output power instead of voltage. Instantaneous power, voltage, current, state of charge (SOC), 
internal temperature, and efficiency are important parameters of the battery which are monitored and calculated by the model developed in one minute interval.

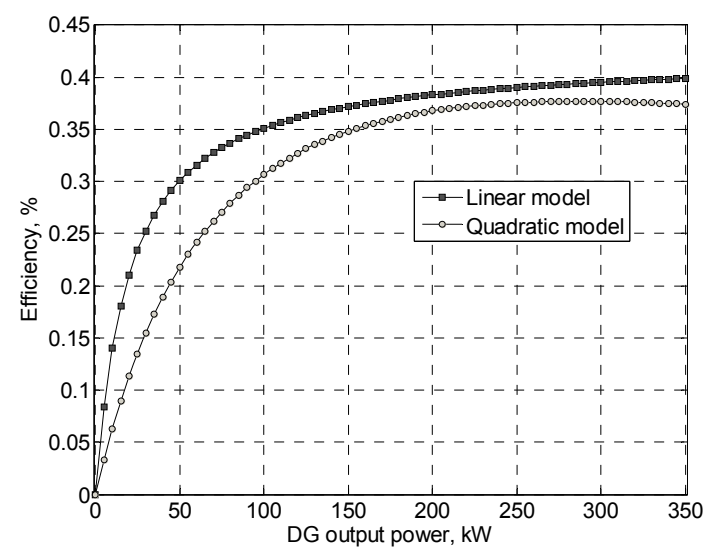

Fig. 2. The overall efficiency of the DiG based on input and output energy.

Battery operational cost, which consists of ownership and operating costs, is also considered in this study. The battery ownership cost (1.1815 \$/Ampere-hour) is calculated based on uncertainty and risk analyses, reported in [21]. Since the battery does not consume fuel and does not experience moving and mechanical parts, its operating cost is treated as a percentage of the total investment cost (1\%-2.5\%) [32].

Battery control is a complicated task in a $\mathrm{MG}$ and is essential for its safe operation. A thorough study of the battery chemical reactions is given in [33], where, the general battery operation framework. A similar principle is utilized for the battery operation in this study, as shown in Fig. 3.

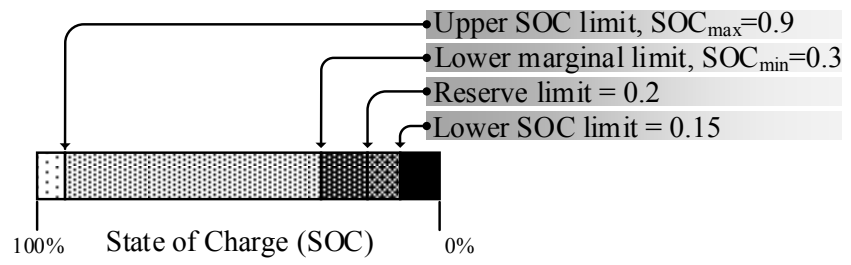

Fig. 3. Classification and operational framework for battery SOC limits [33].

\section{EWH Model}

Residential EWHs are utilized as the DR medium in this study. The dynamic model of a single EWH is similar to the one in [13]. Similar EWH model were also used in [14], [15], where the water temperature is allowed to rise to $160^{\circ} \mathrm{F}$. The thermostatic-controlled valve is utilized in the model to assure safe hot water temperature for the household to avoid scalding and burning. No cost is considered for DR since customers' hot water temperature will not go beyond a pre-defined limit (i.e., customers' quality of service (QoS) is always considered as the highest priority and will not be violated). This procedure only utilizes any excess available energy in the residential EWHs, when available.

Fig. 4 shows a flowchart diagram of the simulation environment developed for this study in MATLAB ${ }^{\circledR}$. At each management interval, the following information (as shown in Fig. 4) are given as input to the simulation environment: hot water flow rate and water temperature of each $\mathrm{EWH}$, ambient temperature and solar irradiation for PV system, nominal DiG capacity, non-EWH load demand, battery SOC, voltage, and temperature from the last interval, and power step value, $\Delta P$. $\Delta P$ is used in each interval to calculate the maximum amount of power to charge the battery (or to discharge from the battery). As shown in Fig. 4, the data will be pre-processed or directly transmitted to the PMA to calculate the operating points of the MG components. Finally, power/energy, cost and emission will be calculated.

A MATLAB ${ }^{\circledR}$-based graphical user interface (GUI) is developed to attain all the required data for the simulation. The GUI allows great flexibility and scalability of the MG for further investigation with different parameters and sizes. Another GUI is also developed to show the real-time simulation results at each interval. At the end of the simulation, a summary of the final results also appears in the GUI.

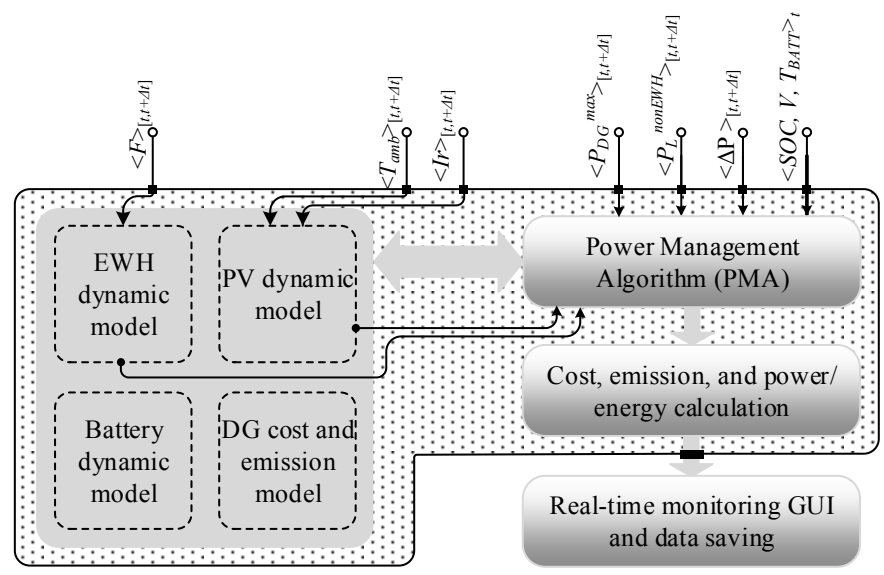

Fig. 4. Flowchart diagram of the MG simulation environment developed for this study.

\section{The Proposed Power Management Algorithm}

MGs often comprise fixed and variable generation, storage, and load within a restricted geographical area. Therefore, it is essential to have a real-time management system to assign appropriate operation point to different components, to protect them from operating outside their safe region, and to manage generation, storage and demand. Numerous objectives have been proposed for MG real-time power management [34]. However, desired objectives may be different from one MG to another, system operators/owners choose the objectives that best fit their MG topology (grid-tied or islanded) to meet their need. In this section, the design of the multi-timescale rulebased power management algorithm for cost-effective operation of the designed MG is explained.

Rule-based power management based on pre-defined rules can be an effective tool for MG operation as long as the number and size of the available components and their dynamic behavior are known to the operator. One major advantage of the rule-based PMA is that they are incredibly fast and computationally efficient compared to the power management algorithms with optimization techniques, particularly in real-time applications. It is because the fact that the decision-making process is already designed based on all possible scenarios using prior knowledge about the system. 
Additionally, rule-based PMAs are easy to implement in realworld applications, particularly for islanded MGs in remote areas.

The multi-timescale simulation and management structure, proposed in this study, helps to improve accuracy and increase the simulation speed by choosing different simulation intervals based on the components' requirements. In this study, simulation for battery and EWHs is carried out every minute, while the PMA is updated every 20 minutes. The simulation interval for $\mathrm{PV}$ and $\mathrm{DiG}$ is the same as the power management interval. The updating interval for the proposed PMA can be changed in the GUI developed for this study. Moreover, the proposed rule-based algorithm targets least cost of the MG operation using least expensive components when possible. Rule-based algorithms become less applicable and effective in MGs with many devices and objectives or MG with features such as plug-and-play operation.

The proposed PMA shown in Fig. 4 is expanded in Fig. 5. As shown in this figure, the battery and EWHs simulations are carried out in one-minute intervals (i.e., dark gray area), while the rest of the simulation (light gray area) is performed in a pre-defined interval, which can be minutes to hours. A description of the input parameters to the PMA is given in Appendix I.

For the non-EWH load demand data, which is available every minute in this study, the average power is calculated and utilized for the length of the management interval. Using all the input parameters shown in Fig. 5, the PMA unit computes the operating point of each MG component. Here, three different rules can be identified (as shown in Fig. 5) which are explained as follows:

RULE I (right column in Fig. 5): In this rule, the total load demand is less than the available PV power in the interval ahead, i.e. $\quad P_{L}^{\text {tot }}<P_{P V}^{\max }$, where $P_{L}^{\text {tot }}=P_{L}^{E W H}+P_{L}^{\text {nonEWH }}$. Therefore, the excess power, i.e. $P_{B A T T}^{s c h}=P_{L}^{t o t}-P_{P V}^{\max }$, can be stored in the battery if the battery SOC is less than the predefined maximum value. The proposed strategy is given in Algorithm 1 of APPENDIX II. Note that battery simulation is performed every minute, regardless of the length of the management interval, to increase the accuracy and better observation of the battery performance. The one-minute simulation interval helps to accurately calculate maximum charging power in the battery considering the minute-byminute battery dynamic and its nonlinear behavior.

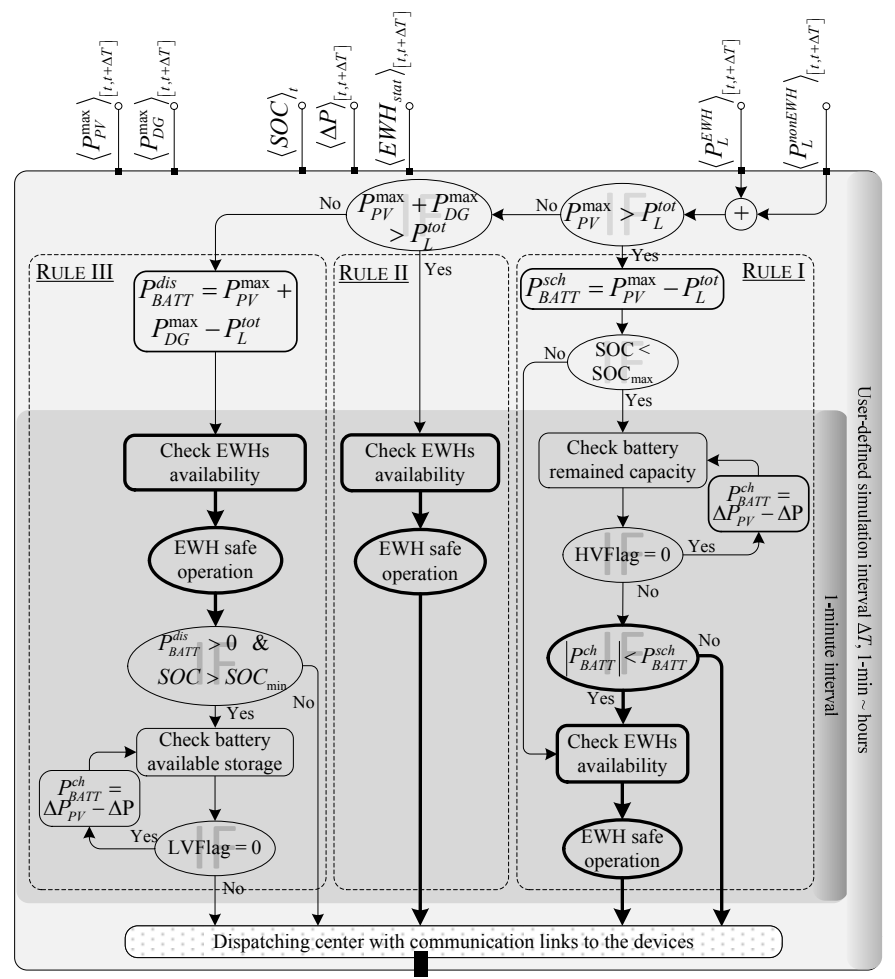

Fig. 5. Schematic diagram for the proposed multi timescale rule-based power management algorithm.

The HVFlag is a variable in the battery model that shows the battery overvoltage or equivalently battery maximum SOC violation, or battery maximum charging power. In RULE I, the battery will be utilized before the DR because a higher battery SOC results in a higher battery lifetime. If the battery is not able to store all the excess PV power, the maximum possible power will be calculated based on the first inner loop shown in the right column in Fig. 5. Based on the convention used in this study, battery is in charging mode when the power is negative $\left(P_{B A T T}^{s c h}<0\right)$, and it is in discharging mode when power is positive $\left(P_{B A T T}^{\text {sch }}>0\right)$. The battery charging algorithm is given in Appendix II. Finally, the operating point of the battery for the interval ahead can be obtained, given as $\left\langle P_{B A T T}\right\rangle_{\left[{ }^{t}, t+\Delta T\right]}$. Since the battery will be charged only by excess $\mathrm{PV}$ generation, no cost is assigned to the battery operation in the charging mode.

If excess PV power is still available (i.e., $\left\langle P_{P V}^{\text {excess }}\right\rangle_{[t, t+\Delta T]}>0$ ), the status of EWHs (as DR resource) will be examined to store the excess power in the form of heat energy. Similar to the battery, this part of the simulation will also be carried out every minute regardless of the management interval. The DR strategies are designed to fulfill two important conditions: 1) the increase in EWHs power should not exceed the excess PV power available at each interval, 2) no more than the prespecified number of EWHs are allowed to be on simultaneously to control peak power. These conditions assure that the EWHs accurately participate in the DR implementation. The proposed DR strategy is given in Algorithm 2 of APPENDIX III. Based on the same principles 
mentioned in Algorithm 2, two different ON/OFF control strategies (explained below) are proposed, where in both cases the customers' quality of service (QoS) has the highest priority:

DR.A: In this case, upper and lower hot water temperature setpoint remain between $120-160{ }^{\circ} \mathrm{F}$, respectively, throughout the day.

DR.B: In this case, a dynamic structure for the upper setpoint for hot water temperature is considered. While the lower setpoint remains at $120{ }^{\circ} \mathrm{F}$ throughout the day for all EWHs, the upper setpoint remains at $130^{\circ} \mathrm{F}$ unless there is excess PV power. In that case, the hot water temperature upper setpoint will be elevated to $160^{\circ} \mathrm{F}$. In DR.A and DR.B when the water temperature goes above $130^{\circ} \mathrm{F}$, the outflowing hot water will be mixed with an appropriate amount of cool water by a thermostatic mixing valve (TMV) prior to use to avoid scalding. Details of the calculations are explained in [14] and [15]. The difference between the two cases (i.e., $D R . A$ and DR.B) is significant, as will be shown in the simulation results.

In the rare cases when there is excess PV power and the battery and EWHs are fully charged, the excess energy needs to be curtailed using power electronic interfaces in the PV system, or dissipates in a dump load.

RULE II (middle column in Fig. 5): In this rule, the total load demand is less than the sum of the available PV power and the DiG nominal power in the interval ahead, i.e., $P_{L}^{\text {tot }}<\left(P_{P V}^{\max }+P_{D i G}^{\max }\right)$. Therefore, the battery is idle, as shown in Fig. 5. However in order to guarantee the cost-effective operation in the proposed PMA, the DR strategy is used to decrease the required power from the $\mathrm{DiG}$, as much as possible, by turning off some of the EWHs. Then, the remaining load is supplied by the DiG, i.e., $\left\langle P_{L}^{t o t}-P_{E W H}^{c t r l}=P_{P V}^{\max }+P_{D i G}\right\rangle_{[t, t+\Delta T]}$, where $P_{E W H}^{c t r l}$ is the amount of

EWH power decreased by the DR strategy. In this study, the $\mathrm{DiG}$ is operated in the standby mode, where it consumes some fuel even at no load. As a result, the total operational cost will be cheaper if the DiG is utilized before the battery. This way, the cost-effective operation of the MG will be achieved, and the MG cost of operation will be equal to the $\mathrm{DiG}$ operational cost. The emissions and damage costs associated with the operation of the DiG will also be calculated in this rule.

RULE III (left column in Fig. 5): In this rule, the total load demand is higher than the total of available PV power and the nominal power of the $\mathrm{DiG}$ in the interval ahead; i.e., $P_{L}^{t o t}>\left(P_{P V}^{\max }+P_{D i G}^{\max }\right)$. Therefore, the battery and DR should be utilized to meet the remaining load demand. Since there is no cost associated with DR in the MG, it is beneficial to perform the DR strategy before the battery. Therefore, the DR procedure will be executed to provide load reduction as much as possible in order to avoid large and random load interruption in the MG. The DR procedure in this rule is similar to the one explained in Algorithm 2 (APPENDIX III) except that the EWHs will be sorted in ascending order based on their hot water temperatures. The two limiting conditions, given for the DR in RULE I, are also valid in this rule. It should be noted that the ON/OFF commands for the EWHs will be overridden in both of the DR algorithms if the hot water temperature goes beyond the pre-defined upper and lower limits. One-minute simulations will always be carried out for the EWHs in this rule.

If there is still undelivered load demand after the DR procedure completed, the battery will be discharged to meet the remaining load demand. In this case, if the battery SOC is higher than the minimum $\left(S O C_{\min }\right)$, the maximum derivable energy from the battery will be calculated for the interval ahead using the inner loop in the left column in Fig. 5. For this purpose, a similar procedure to the one in Algorithm 1 (APPENDIX II) is developed in Algorithm 3 (APPENDIX IV) for the battery discharging, except that the discharged power is positive based on the power flow convention defined in this study. In addition, LVFlag is used instead of the HVFlag, which is introduced in the battery model to show the lowvoltage status of the battery, or equivalently to show if the battery SOC goes below the pre-defined minimum value, or if the pre-defined discharging power limit is violated. High accuracy in the battery model is guaranteed by the one-minute simulation to capture the inherited nonlinearity in the battery model. In rare cases that the battery and the DR cannot meet the shortage in generation, the partial undelivered load in the MG will be realized where some load will be shed. In this rule, MG operational cost will be computed as the sum of DiG cost and the battery cost in the discharging mode.

\section{Simulation Results AND Discussion}

In order to show the effectiveness and applicability of the proposed multi-timescale rule-based power management algorithm, simulation studies using actual data are carried out for the MG presented in Fig. 1. Based on the availability and the type of DR mechanism, three different management algorithms (namely no-DR, DR.A, and DR.B) are simulated and their results are compared. The precision of the calculations is chosen to be $1 \mathrm{~W}$; i.e., all the calculations and actual data are rounded towards $1 \mathrm{~W}$, which is a reasonable assumption for real-world applications.

Since the actual one-minute load data used in this study was only for one house, the load demand data for 100 houses is generated by a series of random numbers with an average of $0.1 \mathrm{~kW}$ and standard deviation of 0.2 , added to the one-house data available to us. The minute-by-minute power consumption of 100 houses is shown in Fig. 6. The different characteristics of the load demand data (e.g., peak hours and annual energy consumption) match the numbers given in [35]. For simulation intervals larger than one minute, the average load demand data is used.

In addition to the non-EWH load data, hot water flow-rate for each customer is also required for aggregate EWH modeling. These data are calculated from the real set of average residential EWH daily demand available in [36] using 
the method explained in [15]. Since there is only average EWH power consumption data available in [36], a technique for conversion of EWH power demand to hot water consumption (flow rate) and a randomization technique, introduced by the authors in [14] and [15], is used to generate hot water demand data for 100 houses, shown in Fig. 7. Normal and uniform distribution is used to randomize the parameters of the EWH model, i.e. tank volume, thermal resistance of tank insulation, initial on/off state, and initial water temperature inside the tank.

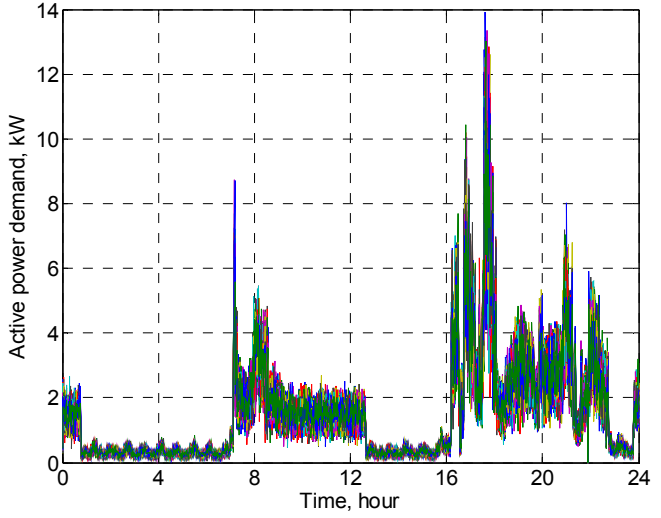

Fig. 6. Minute-by-minute active non-EWH load demand (winter weekday) for 100 houses [17].

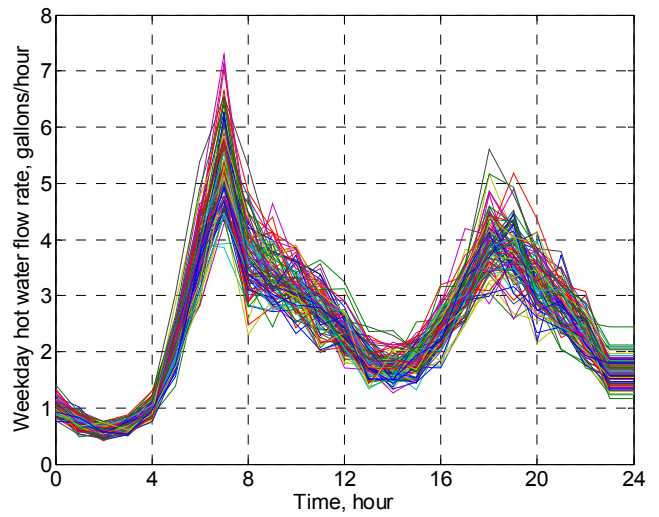

Fig. 7. Hot water consumption for 100 houses in the MG, winter weekday.

The actual temperature and solar irradiation data, employed in this study, are shown in Fig. 8 for a weekday in the winter. It is assumed that all PV modules experience similar ambient temperature and solar irradiation level.

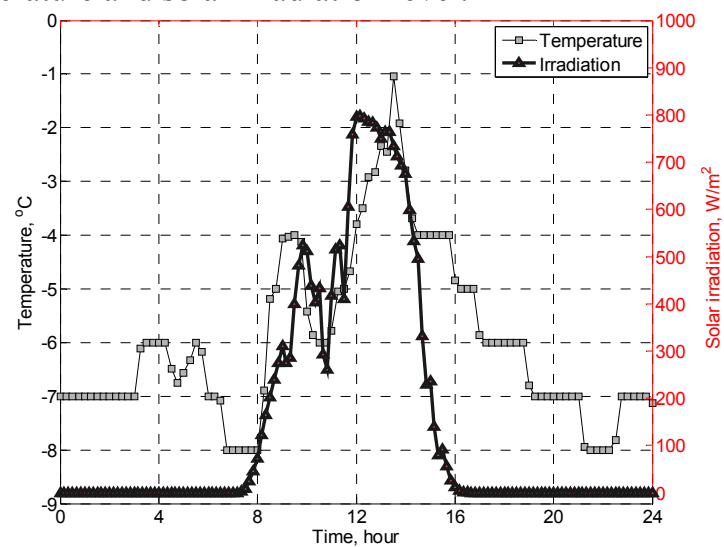

Fig. 8. Temperature and solar irradiation for a non-cloudy weekday in the winter [9].
Three different simulations are performed with $D R . A, D R . B$, and without DR (No-DR) to show the effectiveness of the proposed PMAs with DR. For all simulation studies, the power management is updated every 20 minutes, and the simulation is performed for 24 hours. Additionally, the DiG is operated in standby mode for the three cases. The simulation results are reported in Table III for the MG presented in Fig. 1.

TABLE III

SIMULATION RESULTS OF THE MG OPERATION FOR THE THREE PMAS FOR 24 HOURS.

\begin{tabular}{|c|c|c|c|}
\hline PMAs & $N o-D R$ & DR.A & $D R . B$ \\
\hline \multicolumn{4}{|l|}{ PV } \\
\hline Total used energy, $\mathrm{kWh}$ & 1325.171 & 1631.8417 & 1749.3663 \\
\hline Unused energy, $\mathrm{kWh}$ & 508.4457 & 201.775 & 84.2503 \\
\hline Average efficiency, $\%$ & 89.0605 & 89.7931 & 89.855 \\
\hline \multicolumn{4}{|l|}{$\overline{\mathrm{DiG}}$} \\
\hline Total generation, $\mathrm{kWh}$ & 3187.408 & 2981.8243 & 2876.8337 \\
\hline Fuel Consumption, L & 1044.8309 & 996.173 & 971.1315 \\
\hline Average efficiency, $\%$ & 23.0945 & 22.0459 & 22.0328 \\
\hline \multicolumn{4}{|l|}{ Battery } \\
\hline Discharge, $\mathrm{kWh}$ & 242.645 & 179.2043 & 203.1227 \\
\hline Charge, $\mathrm{kWh}$ & 445.2683 & 440.8677 & 480.9853 \\
\hline Average efficiency, $\%$ & 92.4503 & 92.9302 & 91.1408 \\
\hline \multicolumn{4}{|l|}{ MG Emission } \\
\hline Total $\mathrm{CO}_{2}, \mathrm{~kg}$ & 1661.7 & 1632.4 & 1603.7 \\
\hline Total Non- $\mathrm{CO}_{2}, \mathrm{~kg}$ & 53.4 & 51.0 & 49.9 \\
\hline Total damage cost, $\$$ & 38.2105 & 37.1239 & 36.4132 \\
\hline Total load, kWh & 4309.9557 & 4352.0027 & 4348.3373 \\
\hline Total undelivered load, kWh & 20.7687 & 7.1467 & 8.112 \\
\hline Total MG operation cost, $\$$ & 1339.3116 & 1245.8123 & 1238.1705 \\
\hline
\end{tabular}

In the remainder of this section, the effect of PMAs with $D R . A$ and $D R . B$ on the performance of the $\mathrm{MG}$ and its component is compared with the No-DR PMA, based on the results given in Table III.

SOLAR PV PANEL: It can be seen from Table III that the PV power utilization is increased by $23.1 \%$ and $32.0 \%$ in the $D R . A$ and DR.B algorithms, respectively, compared to the No$D R$. This is because of employing the DR in the management algorithm. When the EWHs' upper thermostat setpoint is increased to $160^{\circ} \mathrm{F}$, a large amount of heat storage capacity is provided by the EWHs hot water tanks. The percentage of increase is less in the DR.A algorithm compared to the DR.B algorithm because the upper thermostat setpoint in the DR.A is set to $160^{\circ} \mathrm{F}$ for the whole day. This way, less free capacity in the hot water tanks will be available when excess PV generation is available in the middle of the day. The increase in power utilization from the PV also resulted in slightly higher efficiency of the PV unit due to higher efficiency of the converters.

DIG: According to Table III, in the PMAs with DR.A and $D R . B$, the total generated energy by the $\mathrm{DiG}$ is decreased by $6.45 \%$ and $9.74 \%$ and the total fuel consumption decreased by $4.66 \%$ and $7.05 \%$, respectively. Since the DiG fuel consumption is a nonlinear function of its output power, the percent reduction in the total generated power by the DiG is not equal to its corresponding total fuel consumption for the two PMAs. Storing the excess PV generation as hot water using the DR strategies (which results in less power 
consumption by the EWHs in the hours ahead) as well as reducing EWHs power consumption during power shortage are the two major reasons for the reduction in the $\mathrm{DiG}$ generation. However, the average efficiency of the DiG is slightly decreased in the DR.A and DR.B algorithms compared to the No-DR algorithm. This is because the utilization of the DR decreases the need for the $\mathrm{DiG}$ power and as a result, the DiG operates in the lower range of its rated power where its efficiency is lower, as shown in Fig. 2.

BATTERY: The battery discharging is decreased by $26.14 \%$ and $16.29 \%$ in DR.A and DR.B, respectively, compared to the No- $D R$ case. This is because using any excess PV generation to preheat water in EWHs decreases the energy required by the EWHs during high demand hours. Therefore, less energy from the battery is required to meet the load demand. In general, any reduction in the battery discharging reduces the MG operational cost, increases battery lifetime, and provides more storage for unexpected loss of PV power and load variations. The battery SOC for the three simulation cases is shown for one day in Fig. 9. The battery discharging starts around 7:30 AM in the DR.B algorithm. Since the upper thermostat setpoint in the DR.B algorithm is set to $130{ }^{\circ} \mathrm{F}$ during normal condition (i.e., during the time of no excess PV generation availability), and because the DR is employed more often, as given in RULE II, more EWHs will turn on early in the morning during first peak period (approximately 7:30-9:00 AM), as shown in Fig. 7. This way, the PV and the DiG cannot meet the total load demand, so the battery is discharged to provide the remaining load demand. The hot water temperature for all households is shown in Fig. 10 (a) and (b) for the DR.A and DR.B PMAs, respectively. It can be seen that at around 7:30 AM, hot water temperatures for most of EWHs reach to about $143^{\circ} \mathrm{F}$ in the PMA with DR.A, and the EWHs stay off during the morning peak hours. However during this peak demand period, the hot water temperature in the majority of the EWHs go below $125^{\circ} \mathrm{F}$ in the DR.B PMA, where some of the EWHs turn on resulting in more demand from the EWHs during the morning peak hours.

In addition, it can be seen from Fig. 9 that the battery discharging occurs approximately during 17:00 to18:30 and 21:00 to $22: 30$ for the No-DR case, while the battery is idle during this period for the other two PMAs. This is because the EWHs could not store hot water during the off-peak hours because of the $130{ }^{\circ} \mathrm{F}$ upper thermostat setpoint limit. Therefore, some of the EWHs will be turned on during the second daily peak hours, which would increase the power demand. As a result, the battery will be discharged to compensate for the power shortage.

Due to the early battery discharge in the DR.B PMA, the accumulative battery charging energy at the end of the day is more than the other PMAs. As shown in Fig. 10 (b), the EWHs hot water temperature increases toward $160^{\circ} \mathrm{F}$ during the excess PV generation hours (approximately during 12:0015:00 hours.) This causes all the EWHs to be off during the second daily peak hours (approximately 16:30-21:00), which is the main reason for lower battery discharging in DR.A and $D R . B$ compared to the No-DR case, shown in Fig. 9. Although the average efficiency of the battery is decreased when the DR is used, the average battery SOC for a day of simulation did not change significantly for the three PMAs. The average battery SOC is $63.8 \%, 64.0 \%$, and $63.3 \%$ for $N o-D R, D R . A$, and $D R . B$, respectively.

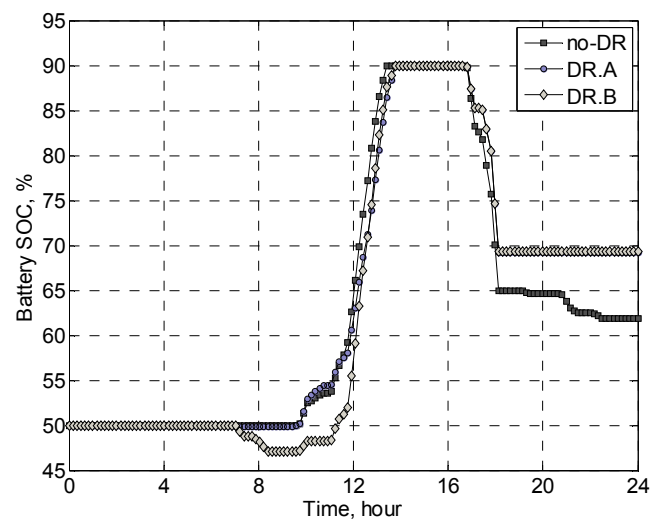

Fig. 9. Battery SOC for the proposed management systems, with and without DR.

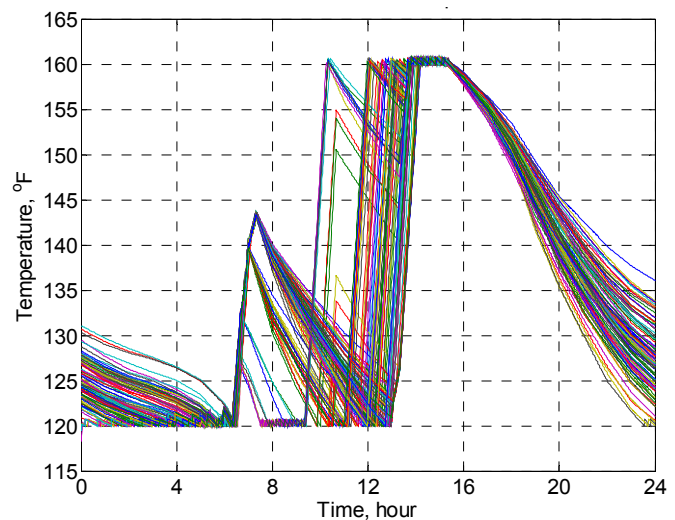

(a)

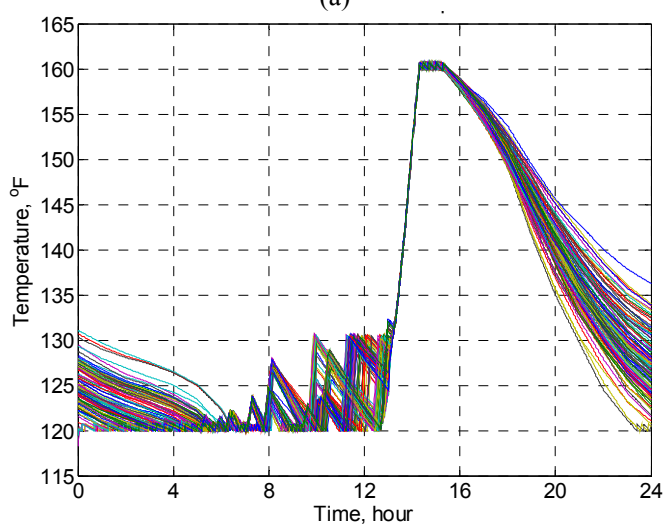

(b)

Fig. 10. The hot water temperature for all household in (a) DR.A, and (b) DR.B PMAs.

MG EMISSIONS: As expected, the total MG emission is reduced because of the $\mathrm{DiG}$ generation reduction when the DR strategies are used. The reduction in $\mathrm{CO}_{2}$ and non- $\mathrm{CO}_{2}$ emissions for the DR.A and the DR.B compared to the No-DR PMA are tabulated in Table IV. $1.8 \%$ and $3.5 \%$ reduction for $\mathrm{CO}_{2}$ emission as well as $4.5 \%$ and $6.6 \%$ reduction in non- $\mathrm{CO}_{2}$ emissions are observed for the DR.A and DR.B, respectively, compared to the $N o-D R$ case. These reductions in emission resulted in $2.8 \%$ and $4.7 \%$ decrease in the total damage cost 
for $D R . A$ and $D R . B$, respectively, compared to the $N o-D R$ case.

TABLE IV

REDUCTION IN $\mathrm{CO}_{2}$ AND NON-CO $\mathrm{CO}_{2}$ EMISSIONS IN THE $D R . A$ AND $D R . B$ PMAS

\begin{tabular}{llccc}
\multicolumn{1}{c}{ PMAs } & & DR.A & & DR.B \\
\cline { 1 - 1 } \cline { 5 - 6 } Decrease in $\mathrm{CO}_{2}$ emissions & & $1.8 \%$ & & $3.5 \%$ \\
Decrease in non- $\mathrm{CO}_{2}$ emissions & & $4.5 \%$ & & $6.6 \%$ \\
\hline
\end{tabular}

TOTAL UNDELIVERED LOAD: Although for the size of the MG studied, the total undelivered power for the No-DR PMA is negligible (less than $0.5 \%$ compared to the total load demand), this value is reduced by $65.6 \%$ and $60.9 \%$ for the DR.A and $D R . B$ PMAs, respectively. Undelivered power occurs when the total available generation, storage, and reduction in EWHs' power is less than the sum of the non-EWH load demand and the power demand of the EWHs that are forced to be on. For the DR.A and DR.B PMAs, undelivered load demand occurred during 16:40-17:00 (1.659 kW and 1.329 $\mathrm{kW}$ for $D R . A$ and $D R . B$, respectively) and during 17:40-18:00 $(19.781 \mathrm{~kW}$ and $22.998 \mathrm{~kW}$ for DR.A and DR.B, respectively). According to Fig. 10, all EWHs are off during these periods, and the DR cannot be used to avoid undelivered load demand.

Total MG Operational Cost: As given in Table III, the total MG operational cost is reduced by $6.98 \%$ and $7.55 \%$ for the DR.A and DR.B PMAs, respectively, compared to the No$D R$ PMA. It is because of the fact that when the DR strategies are employed, the DiG output energy and the battery discharge decrease. These reductions in energy demand have resulted in noticeable cost reduction in the MG operation.

\section{CONCLUSION}

In this paper, a rule-based multi-timescale cost-effective power management algorithm is proposed to effectively include generation, storage, and the DR within the same management framework. The MG design and comprehensive modeling are briefly discussed, and the power management algorithm is presented. Two different EWH DR strategies (DR.A and DR.B) are proposed and compared with the No-DR case. Simulation results showed that incorporating the DR in the management algorithm increased the PV power utilization; decreased the DiG total generation, its fuel consumption and emissions; increased the battery average SOC; and decreased the total undelivered load in the MG. As a result, the MG operational costs are decreased remarkably when the DR is employed in the PMA.

Each of the DR strategies has certain advantages over the other; the choice of which one to use depends on the specific circumstances and is to be made by the owner/operator of the MG. DR.A is a better approach when the average efficiency of the DiG and battery, and the undelivered load demand are important. On the other hand, DR.B is better in terms of the MG operational cost, PV power utilization, and the total MG emissions. It should be noted that the proposed PMAs need to be modified to accommodate other variable generation sources (such as wind) according to their variable nature, as was done in this paper for solar PV.

\section{APPENDIX I}

$\checkmark\left\langle P_{P V}^{\max }\right\rangle_{[t, t+\Delta T]}:$ Maximum PV available power for the interval ahead (i.e., $t$ to $t+\Delta T$ ), calculated by "comprehensive PV model" module, shown in Fig. 4.

$\checkmark\left\langle P_{D i G}^{\max }\right\rangle_{[t, t+\Delta T]}$ : Nominal capacity of the DiG unit. $P_{D i G}$ is a constant throughout this paper, but it can change in different time intervals.

$\checkmark\langle S O C\rangle_{t}$ : The latest value of the battery SOC.

$\checkmark\langle\Delta P\rangle_{[t, t+\Delta T]}$ : is used in each interval to calculate the maximum amount of power to charge the battery (or to discharge from the battery).

$\checkmark\left\langle E W H_{\text {stat }}\right\rangle_{[t, t+\Delta T]}$ : EWHs' primary ON/OFF status calculated by "EWH dynamic model" module, shown in Fig. 4, based on the actual hot water flow rate at the beginning of the simulation for each interval.

$\checkmark\left\langle P_{L}^{E W H}\right\rangle_{[t, t+\Delta T]}$ : Load demand from the EWHs for $t$ to $t+\Delta T$ interval, calculated by "EWH dynamic model" module.

$\checkmark\left\langle P_{L}^{\text {nonEWH }}\right\rangle_{[t, t+\Delta T]}$ : Non-EWH load demand for $t$ to $t+\Delta T$ interval.

\section{APPENDIX II}

\section{Algorithm 1. BATTERY CHARGING}

Required: Battery SOC, internal voltage and temperature at the end of the last interval, step power change $(\Delta P)$, battery scheduled power $\left(P_{B A T T}^{s c h}\right)$, and simulation interval $(\Delta T)$.

1: $P_{B A T T}^{c h} \leftarrow P_{B A T T}^{s c h}$

2: $\quad$ HVFlag $=$ BattModel $^{\dagger}\left(P_{B A T T}^{c h},\langle S O C, V, T\rangle_{t}, \Delta T\right)$

3: While $($ HVFlag $=0) \&\left(P_{B A T T}^{c h}<0\right)$ do

4: $\quad P_{B A T T}^{c h}=P_{B A T T}^{c h}+\Delta P$

5: $\quad$ HVFlag $=\operatorname{BattModel}\left(P_{B A T T}^{c h},\langle S O C, V, T\rangle_{t}, \Delta T\right)$

6: End While

7: If $($ HVFlag $=1) \&\left(P_{B A T T}^{c h}<0\right)$ then

8: $\left\langle P_{B A T T}\right\rangle_{[t, t+\Delta T]}=P_{B A T T}^{c h} ;\left\langle P_{P V}^{\text {excess }}\right\rangle_{[t, t+\Delta T]}=P_{B A T T}^{s c h}-P_{B A T T}^{c h} ;$

9: Else

10: $\left\langle P_{B A T T}\right\rangle_{[t, t+\Delta T]}=0 ; \quad\left\langle P_{P V}^{\text {excess }}\right\rangle_{[t, t+\Delta T]}=P_{B A T T}^{s c h} ;$

11: End If

"BattModel" is the dynamic model of the battery developed in MATLAB $^{\circledR}$ m-file.

APPENDIX III

Algorithm 2. EWHS ON/OFF CONTROL AlgORITHM* 
Required: PV excess power ( $\left.P_{P V}^{\text {excess }}\right)$, EWHs consumption and status $\left(S_{i}^{t+\Delta T}\right)$ for the interval ahead and at the end of the last interval, number of EWHs ( $\left.N O_{E W H}\right)$, and simulation interval $(\Delta T)$.

1: $P_{D R}^{o n} \leftarrow P_{P V}^{\text {excess }}, t \leftarrow 1$

2: For $(\mathrm{t}<\Delta T)$ do

3: $\quad$ Ind $\boldsymbol{s o r t}^{\dagger}\left(\left\langle T_{i}\right\rangle_{t} ; i=1, \ldots, N O_{E W H}\right.$, 'descending' $)$

4: $\quad$ calculate $P_{E W H}^{\text {total, orig }}(t)$,

5: While $\left(\mathrm{i}<N O_{E W H}\right)$ do

6: $\quad$ If $\left(\left\langle T_{i}\right\rangle_{t-1}>T_{i}^{\max }\right)$ then $S_{i}(t) \leftarrow 0$

7: $\quad$ Else If $\left(\left\langle T_{i}\right\rangle_{t-1}<T_{i}^{\text {min }}\right)$ then $S_{i}(t) \leftarrow 1$

8: $\quad$ Else

9: $\quad$ If $\left(S_{i}(t)=0\right)$, then $S_{i}(t)=1$

10: $\quad$ temp $=\operatorname{sum}\left(S_{i}(t) ; i=1, \ldots, N O_{E W H}\right)$

11: $\quad$ calculate $P_{E W H}^{\text {total }, \text { new }}(t)$

11: If (temp $>$ Max $\left._{\text {on }}\right) \|$

$$
\left(\left(P_{E W H}^{\text {total, new }}(t)-P_{E W H}^{\text {total,orig }}(t)\right)>P_{D R}^{o n}\right) \text {, then }
$$

12:

$$
S_{i}(t)=0 ; \text { break; }
$$

13:

14:

15:

\section{End If}

\section{End If}

End If

16 :

17:

18: End While

19: $t \leftarrow t+1$

\section{0: End For}

21: $\left\langle P_{P V}^{\text {excess }}=P_{P V}^{\text {excess }}-\left(P_{E W H}^{\text {total, new }}(t)-P_{E W H}^{\text {total, orig }}(t)\right)\right\rangle_{[t, t+\Delta T]}$

$\dagger$ "sort" is the sorting algorithm in MATLAB ${ }^{\circledR}$.

\$ "EWHModel" is the dynamic model of the single EWH developed in MATLAB $^{\circledR}$ m-file.

* Required modifications for Algorithm 2 in RULE III for reduction in the EWHs consumption:

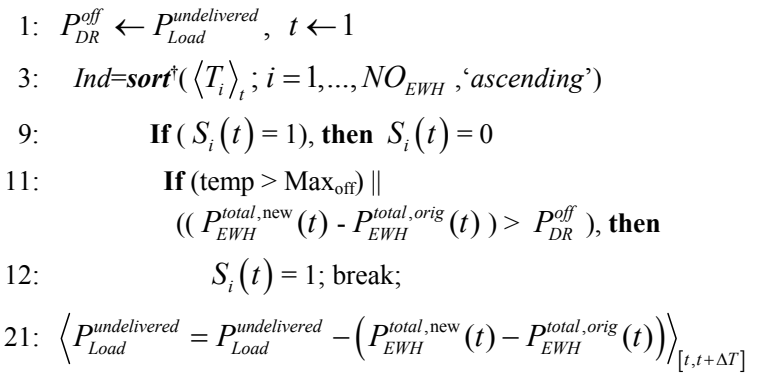

\section{APPENDIX IV}

\section{Algorithm 3. BATTERY DISCHARGING}

Required: Battery SOC, internal voltage and temperature at the end of the last interval, step power change $(\Delta P)$, battery scheduled power $\left(P_{B A T T}^{s c h}\right)$, and simulation interval $(\Delta T)$.

1: $P_{B A T T}^{d i s} \leftarrow P_{B A T T}^{s c h}$

2: $\quad$ LVFlag $=$ BattModel $^{\dagger}\left(P_{B A T T}^{d i s},\langle S O C, V, T\rangle_{t}, \Delta T\right)$

3: While $($ LVFlag $=0) \&\left(P_{B A T T}^{d i s}>0\right)$ do
4: $\quad P_{B A T T}^{d i s}=P_{B A T T}^{d i s}-\Delta P$

5: $\quad$ LVFlag $=\operatorname{BattModel}\left(P_{B A T T}^{d i s},\langle S O C, V, T\rangle_{t}, \Delta T\right)$

6: End While

7: If $($ LVFlag $=1) \&\left(P_{B A T T}^{d i s}>0\right)$ then

8: $\left\langle P_{B A T T}\right\rangle_{[t, t+\Delta T]}=P_{B A T T}^{\text {dis }} ;\left\langle P_{\text {Load }}^{\text {undelivered }}\right\rangle_{[t, t+\Delta T]}=P_{B A T T}^{s c h}-P_{B A T T}^{\text {dis }} ;$

9: Else

10: $\left\langle P_{B A T T}\right\rangle_{[t, t+\Delta T]}=0 ; \quad\left\langle P_{\text {Load }}^{\text {undelivered }}\right\rangle_{[t, t+\Delta T]}=P_{B A T T}^{s c h} ;$

11: End If

$\dagger$ "BattModel" is the dynamic model of the battery developed in MATLAB $^{\circledR}$ m-file.

\section{REFERENCES}

[1] U.S. DOE, office of electric transmission and distribution, "GRID 2030: A national vision for electricity's second 100 years," July 2003.

[2] F. Katiraei, R. Iravani, N. Hatziargyriou, and A. Dimeas, "Microgrids management: controls and operation aspects of microgrids," IEEE Power Energy Mag., vol. 6, no. 3, 2008, pp. 54-65.

[3] C. M. Colson, M. H. Nehrir, and S. A. Pourmousavi, "Towards real-time microgrid power management using computational intelligence methods," in Proc. PES GM, Minneapolis, USA 2010, pp. 1-8.

[4] A. G. Tsikalakis, and N. D. Hatziargyriou, "Centralized control for optimizing microgrids operation," IEEE Trans. Energy Convers., vol. 23, no. 1, pp. 241-248, Feb. 2008.

[5] S. A. Pourmousavi, M. H. Nehrir, C. M. Colson, and C. Wang, "Realtime energy management of a stand-alone hybrid wind-microturbine energy system using particle swarm optimization," IEEE Trans. Sustain. Energy, vol. 1, no 3, 2010, pp. 193-201.

[6] F. A. Mohamed and H. N. Kovio, "Power management strategy for solving power dispatch problems in Microgrid for residential applications," in Proc. IEEE International Energy Conference and Exhibition, Manama, Bahrain 2010, pp. 746-751.

[7] Y. Riffonneau, S. Bacha, F. Barruel, S. Ploix, "Optimal power flow management for grid connected PV systems with batteries," IEEE Trans. Sustain. Energy, vol. 2, no. 3, pp. 309-320, Feb. 2011.

[8] B. Belvedere, et. Al., "A microcontroller-based power management system for standalone microgrids with hybrid power supply," IEEE Trans. Smart Grid, vol. 3, no. 3, pp. 422-431, May 2012.

[9] S. A. Pourmousavi, R. K. Sharma, and B. Asghari, "A framework for real-time power management of a grid-tied microgrid to extend battery lifetime and reduce cost of energy," in Proc. IEEE PES Innovative Smart Grid Technologies, Washington D.C., USA 2012, pp. 1-8.

[10] C. M. Colson and M. H. Nehrir, "Comprehensive real-time microgrid power management and control with distributed agents," IEEE Trans. Smart Grid, vol. 4, no. 1, pp. 617-627, Jan. 2013.

[11] C. Colson, M. H. Nehrir, R. K. Sharma, and B. Asghari, "Improving sustainability of hybrid energy systems Part II: Managing multiple objectives with a multiagent system," IEEE Trans. Sustain. Energy, vol. 5, no. 1, pp. 46-54, Jan. 2014.

[12] C. Colson, M. H. Nehrir, R. K. Sharma, and B. Asghari, "Improving sustainability of hybrid energy systems Part I: Incorporating battery round-trip efficiency and operational cost factors," IEEE Trans. Sustain. Energy, vol. 5, no. 1, pp. 37-45, Jan. 2014.

[13] P.S. Dolan, M.H. Nehrir, V. Gerez, "Development of a Monte Carlo based aggregate model for residential electric water heater loads," Electr. Pow. Syst. Res., vol. 36, no. 1, pp. 29-35, Jan. 1996.

[14] S. N. Patrick, "Control of Aggregate Electric Water Heaters for Load Shifting and Balancing Intermittent Renewable Energy Generation in a Smart Grid Environment," M.S thesis in Electrical Engineering, Montana State University-Bozeman, 2011.

[15] S. A. Pourmousavi, S. N. Patrick, M. H. Nehrir, "Real-time demand response through aggregate electric water heaters for load shifting and balancing intermittent wind generation," IEEE Transactions on Smart Grid, vol. 5, no. 2, pp. 769-778, March 2014.

[16] HOMER ENERGY: Energy Modeling Software for Hybrid Renewable Energy Systems, Available online at: http://homerenergy.com/ 
[17] "Individual household electric power consumption Data Set," Machine Learning Repository, University of California-Irvine, available online: http://archive.ics.uci.edu/ml/datasets/Individual+household + electric + po wer+consumption\#

[18] E. Lorenzo, et. Al, Solar Electricity Engineering of Photovoltaic Systems, Earthscan Publications Ltd., pp. 316, Jan. 1994.

[19] SunPower Corp., "SPR-200-BLK, residential PV Module," specification sheet available online: http://www.energyplus.com.sg/products/32.pdf

[20] L.E. Bussey, The Economic Analysis of Industrial Projects, Prentic-Hall Inc.: New Jersey, 1978, ch. 4 and 11.

[21] S. A. Pourmousavi, M. H. Nehrir, and R. K. Sharma, "Ownership cost calculations for distributed energy resources using uncertainty and risk analysis," Submitted to the IEEE Trans. Pow. Sys. for possible publication, March 2014.

[22] S. Chakraborty and M. G. Simoes, "PV-microgrid operational cost minimization by neural forecasting and heuristic optimization," in Proc. IEEE Industry Applications Society Annual Meeting, Edmonton, Canada 2008, pp. 1-8.

[23] CumminsOnan Commercial SD 10000, Available online: https://powersuite.cummins.com/PSWEB/login.action

[24] M. Pipattanasomporn, M. Willingham, S. Rahman, "Implications of onsite distributed generation for commercial/industrial facilities," IEEE Trans. Power Syst., vol. 20, no. 1, pp. 206-212, Feb 2005.

[25] MATLAB Curve Fitting Toolbox, MathWorks Inc., Available online at: http://www.mathworks.com/products/curvefitting/

[26] A. Sobu and G. Wu, "Dynamic optimal schedule management method for microgrid system considering forecast errors of renewable power generations," in Proc. IEEE International Conference on Power system Technology, Auckland, USA 2012, pp. 1-6.

[27] D. Thimsen, "Cost of utility distributed generators, 1-10 MW," EPRI, Rep. no. 1007760, March 2003.

[28] D. Zhu, et. Al., "In-plume emission test stand 2: Emission factors for 10to 100-kW U.S. Military Generators," JAPCA J. Air Waste Ma., vol. 59, no. 12, pp. 1446-1457, Dec. 2009.

[29] R. S. J. Tol, "The marginal damage costs of carbon dioxide emissions: an assessment of the uncertainties," Energ. Policy, vol. 33, no. 16, pp. 2064-2074, Nov. 2005.

[30] D. Burtraw, S.J. Szambelan, "U.S. emissions trading markets for $\mathrm{SO}_{2}$ and $\mathrm{NO}_{\mathrm{x}}$," Discussion Paper, Resources for the future company, RFF DP 09-40, Oct. 2009.

[31] M. Ceraolo, "New dynamical models of lead-acid batteries," IEEE Trans. Power Syst., vol. 15, no. 4, pp. 1184-1190, Nov. 2000.

[32] EPRI, "Electricity Energy Storage Technology Options: A White Paper Primer on Applications, Costs and Benefits," Dec, 2010. Web Address: http://www.electricitystorage.org/images/uploads/static_content/technol ogy/resources/ESA_TR_5_11_EPRIStorageReport_Rastler.pdf.

[33] R. Lassseter, M. Erikson, "Integration of battery-based energy storage element in the CERTS microgrid," Final Report Task 4, prepared for U.S. DOE., Oct. 2009, ch. 6.

[34] C. M. Colson, and M. H. Nehrir, "A review of challenges to real-time power management of microgrids," in Proc. IEEE PES GM, Calgary, Canada 2009, pp. 1-8.

[35] C. M. Colson, and M. H. Nehrir, "An alternative method to load modeling for obtaining end-use load profiles," in Proc. IEEE NAPS, Starkville, USA 2009, pp. 1-5.

[36] "Descriptions of electric energy use in single family residences in the pacific northwest, 1986- 1992," Office of Energy Resources, Bonneville Power Administration, Portland, OR, December 1992.

\section{BIOGRAPHIES}

S. Ali Pourmousavi received his Ph.D. in the Electrical and Computer Engineering (ECE) Department at Montana State University-Bozeman, MT in 2014. Currently he is a post doc fellow at NEC-Labs America.

M. Hashem Nehrir is a professor in the Electrical and Computer Engineering Department at Montana State University- Bozeman.

Ratnesh K. Sharma is the Founding Head of the Energy Management Department, NEC Laboratories America, Princeton, NJ, USA. 